\title{
Clinical utility gene card for oculocutaneous (OCA) and ocular albinism (OA)—an update
}

\author{
Abdullah Aamir ${ }^{1} \cdot$ Helen J. Kuht ${ }^{1} \cdot$ Karen Grønskov $\mathbb{D}^{2} \cdot$ Brian P. Brooks $^{3} \cdot$ Mervyn G. Thomas $\mathbb{D}^{1}$
}

Received: 26 August 2020 / Revised: 12 December 2020 / Accepted: 8 January 2021 / Published online: 27 January 2021

(c) The Author(s), under exclusive licence to European Society of Human Genetics 2021

\section{Disease characteristics}

\subsection{Name of the disease (synonyms)}

Oculocutaneous albinism (OCA). Subtypes include OCA1 (OCA1A and OCA1B), OCA2, OCA3, OCA4, OCA5, OCA6 and OCA7.

Ocular albinism (OA). Synonyms include NettleshipFalls Ocular Albinism, Ocular Albinism Type 1 (OA1), XLinked Ocular Albinism (XLOA).

\subsection{OMIM\# of the disease}

Table 1 summarises the disease subtypes and associated OMIM number.

\subsection{Name of the analysed genes or DNA/ chromosome segments}

OCA: TYR, OCA2, TYRP1, SLC45A2, LRMDA (C10orf11) and $S L C 24 A 5$.

OA: GPR143

\subsection{OMIM\# of the gene(s)}

Table 2 summarises the genes with associated OMIM number.

Mervyn G. Thomas

mt350@le.ac.uk

1 The University of Leicester Ulverscroft Eye Unit, Department of Neuroscience, Psychology and Behaviour, University of Leicester, RKCSB, PO Box 65, Leicester LE2 7LX, UK

2 Rigshospitalet, Department of Clinical Genetics, Kennedy Center, Copenhagen University Hospital, Glostrup, Denmark

3 Ophthalmic Genetics and Visual Function Branch, National Eye Institute, Bethesda, MD, USA
Review of the analytical and clinical validity as well as of the clinical utility of DNA-based testing for variants in the albinism genes in diagnostic and prenatal settings and for risk assessment in relatives.

\subsection{Mutational spectrum}

Oculocutaneous albinism (OCA) describes a group of inherited (autosomal recessive) conditions which are characterised by disruption to the melanin biosynthesis pathway resulting in cutaneous and ocular hypopigmentation and visual deficits. OCA can be subdivided into 7 different types, dependent on the type of genetic variant. Most genetic variants associated with OCA include missense, nonsense or frameshifts. Larger deletions or duplications are less frequently reported. The HGMD professional database (version 2020.2) lists 464 variants affecting function in TYR, 342 in OCA2, 51 in TYRP1, 163 in SLC45A2, 28 in SLC24A5, 6 in LRMDA and 192 in GPR143. The Leiden Open Variant Database (LOVD) and ClinVar are freely accessible databases also providing information on variants in the above genes. Further mutational spectrum details associated with each OCA type are as follows (in order of most commonly occurring in a European cohort):

(OCA1) TYR: missense, nonsense, splice, deletion and insertion variants, exon and whole gene deletions. Approximately $42 \%$ of cases may be attributable to variants affecting function in TYR [1]. The impact of two variants, (NM_000372.4) c.575C $>$ A, p.(Ser192Tyr) (S192Y) and c.1205G $>$ A, p.(Arg402Gln) (R402Q), on protein function and the albinism phenotype has been widely debated. Functional studies have demonstrated that both of these variants result in reduced functional activity of the tyrosinase enzyme [2], yet in isolation they may not result in albinism [3]. However, it has been reported that a diseasecausing variant inherited with both of these alleles in cis may reduce function enough to result in a hypomorphic phenotype [4]. In a European population with similar hypomorphic features, a further haplotype has been 
Table 1 OCA subtype with corresponding phenotype MIM number.

\begin{tabular}{ll}
\hline OCA subtype & Phenotype MIM number \\
\hline OCA1A & 203100 \\
OCA1B & 606952 \\
OCA2 & 203200 \\
OCA3 & 203290 \\
OCA4 & 606574 \\
OCA5 & 615312 \\
OCA6 & 113750 \\
OCA7 & 615179 \\
OA & 300500
\end{tabular}

Table 2 OCA/OA gene with corresponding OMIM gene number.

\begin{tabular}{ll}
\hline Gene & OMIM $\sharp$ (gene) \\
\hline TYR & 606933 \\
OCA2 & 611409 \\
TYRP1 & 115501 \\
SLC45A2 & 606202 \\
LRMDA (C10orf11) & 614537 \\
SLC24A5 & 609802 \\
GPR143 & 300808 \\
\hline
\end{tabular}

described, containing both S192Y and R402Q alleles [5]. Other studies that identified cases with both a $T Y R$ variant affecting function and R402Q (in trans) or a $T Y R$ variant affecting function combined with R402Q/S192Y in cis do not find an additive effect of the S192Y variant as previously suggested $[1,6]$.

(OCA2) OCA2: missense, nonsense, splice, deletions and insertion variants, exon and whole gene deletions and duplications [7]. Homozygous recessive variants in $O C A 2$ are found as a cause in $28 \%$ of suspected cases [1].

(OCA3) TYRP1: missense, nonsense, deletions and insertion variants [8]. OCA3 accounts for $2 \%$ of cases [1].

(OCA4) SLC45A2: missense, nonsense, splice, deletion and insertion variants, exon and whole gene deletions and duplications [9]. Approximately $10.5 \%$ of cases are due to variants in SLC45A2 [1].

(OCA6) SLC24A5: missense, nonsense, splice, deletion and insertion variants and exon deletion [10]. Variants affecting function in SLC24A5 are found in $23 \%$ of cases [1].

(OCA7) LRMDA: nonsense and frameshift variants $[11,12]$.

Ocular albinism is an X-linked disorder resembling the features of OCA without distinct cutaneous hypopigmentation. Obligatory female carriers have been described as harbouring a "mud-splattered" fundus appearance due to some hypopigmentation, but rarely manifest with visual impairment [13].
(OA1) GPR143: missense, nonsense, splice, deletion, insertion and duplication [14].

There has been one case report [15] of co-existing recessive variants in GPR143 (ocular albinism) and TYRP1 (OCA3).

\subsection{Analytical methods}

Due to the genetic heterogeneity of OCA, next-generation sequencing (NGS) approaches comprising multigene panels or whole-exome sequencing (WES) are being increasingly used and our group has previously reported the utility of a novel gene panel using NGS $[1,16,17]$. Sanger sequencing is used for segregation analysis and validation of variants identified from NGS. Gene targeted testing using multiplex ligation-dependent probe amplification (MLPA), comparative genomic hybridisation (CGH) and quantitative PCR (qPCR) may be used to detect large deletions or duplications [18].

\subsection{Analytical validation}

Sanger sequencing is helpful for validating variants identified using NGS and also performing segregation in the parents of an index case, thus allowing for confirmation of variants existing in trans. Functional analysis combined with in silico variant prediction are useful in validating novel variants [9].

\subsection{Estimated frequency of the disease}

(Incidence at birth ("birth prevalence") or population prevalence. If known to be variable between ethnic groups, please report):

In the general population, estimated frequency is $\sim 1$ in 20,000 [19]. In sub-Saharan Africa, reported frequencies are approximately between 1 in 4000 and 1 in 1000 [20]. While it is deemed that the reported frequencies are lower in Caucasian populations due to mild cases appearing less prominent amongst an already light-skinned demographic, recent work [21] has estimated a frequency amongst the Dutch population of at least 1 in 12,000 accounting for all genetic forms of albinism. Reports have estimated the point prevalence of OA1 to be around 1 in 60,000 in a Danish cohort [22]. Table 3 summarises estimated frequencies by type of OCA.

Variants in SLC24A5 (OCA6) have been reported in 33 patients in the literature, including European, African and Asian ethnicities [10, 26]. Variants in LRMDA (OCA7) have been reported in few patients from the Faroe Islands, Lithuania and Arabian Peninsula [1]. LRMDA is the rarest identified genetic subtype of albinism with no data on prevalence. The 4q24 locus (OCA5) has been mapped in a 
Table 3 Estimated population prevalence by OCA type.

\begin{tabular}{ll}
\hline OCA type & Estimated frequency \\
\hline OCA1 & 1 in 40,000 [23] \\
OCA2 & 1 in 36,000 (White Europeans) \\
& 1 in 3900 (Africans) [24] \\
OCA3 & 1 in 8500 (Africans) [25] \\
OCA4 & 1 in 85,000 (Japanese) [8] \\
OA1 & 1 in 60,000 (Europeans) \\
\hline
\end{tabular}

consanguineous family from Pakistan, but no gene has yet been identified [27].

\subsection{Diagnostic setting}

\begin{tabular}{lll} 
& Yes. & No. \\
A. (Differential) diagnosis & $\bigotimes$ & $\square$ \\
B. Predictive Testing & $\square$ & $\square$ \\
C. Risk assessment in Relatives & $\bigotimes$ & $\square$ \\
D. Prenatal & $\bigotimes$ & $\square$ \\
\hline
\end{tabular}

Comment: Variant analysis aids in the confirmation of a clinical diagnosis and subsequent genetic counselling. There are some reports of prenatal investigations in families with confirmed OCA in the literature [28, 29], using both chorionic villus sampling and amniocentesis.

\section{Test characteristics}

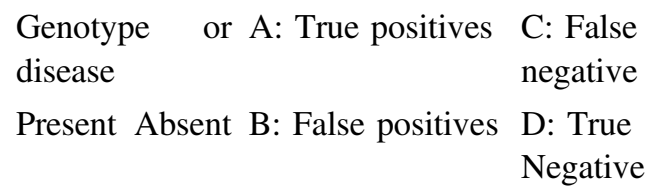

Test

$\begin{array}{llll}\text { Pos. A } & \text { B } & \text { Sensitivity: } & \mathrm{A} /(\mathrm{A}+\mathrm{C}) \\ & & \text { Specificity: } & \mathrm{D} /(\mathrm{D}+\mathrm{B}) \\ \text { Neg. C } & \text { D } & \text { Pos. } & \mathrm{A} /(\mathrm{A}+\mathrm{B}) \\ & & \text { predict. value: } & \mathrm{D} /(\mathrm{C}+\mathrm{D}) \\ & & \\ & & \text { Neg. } \\ & \text { predict. value: } & \\ & & \end{array}$

\subsection{Analytical sensitivity (proportion of positive tests if the genotype is present)}

If the genotype is present NGS and Sanger sequencing would detect most variants $(<100 \%)$, however deep intronic variants and copy number variants could potentially be missed. Thus, NGS with coverage of intronic regions together with a supplementary copy number diagnostic test would have higher analytical sensitivity. Moreover, the relevance of coverage of deep intronic regions have been highlighted by identification of the pathogenic TYR haplotype [5] and TYR upstream variants [17]. Sensitivity of $>90 \%$ has been reported when using such an approach in patients with suspected albinism [30].

\subsection{Analytical specificity (proportion of negative tests if the genotype is not present)}

NGS captures multiple genes associated with albinism therefore has a risk of misinterpretation of rare variants and hence false positives. Thus, resulting in a $<100 \%$ analytical specificity. Interpretation of variants within the clinical and biological context reduces the risk of misinterpretation of rare variants.

\subsection{Clinical sensitivity (proportion of positive tests if the disease is present)}

Various factors such as family history and age may affect the clinical sensitivity. Reported detection rates for OCA1A, OCA1B are $75-90 \%$ and $37 \%$, respectively. Often patients with phenotypical features of OCA are only identified to have one variant. However, recent work has identified a haplotype (NC_000011.10:g.89278565A $>\mathrm{G}$ and NC_000011.10:g.89078081C $>$ G) that is significantly enriched in patients with only one identified variant affecting function in TYR, particularly those with $\mathrm{R} 402 \mathrm{Q} / \mathrm{S} 192 \mathrm{Y}$ as described previously, therefore uncovering previously missing heritability in mild cases of albinism [5].

\subsection{Clinical specificity (proportion of negative tests if the disease is not present)}

The clinical specificity can be dependent on variable factors such as age or family history. In such cases, a general statement should be given, even if a quantification can only be made case by case.

A number of polymorphisms and variants of unknown significance exist within genes implicated in OCA, which may be contributing to variance in pigmentation, particularly in an already lightly pigmented population.

\subsection{Positive clinical predictive value (life-time risk to develop the disease if the test is positive)}

For OCA the disease is thought to be fully penetrant, although expressivity varies.

With a GPR143 variant affecting function (OA), the lifetime risk is $100 \%$ in males. However heterozygous 
female carriers can exhibit carrier signs including iris transillumination defects, mud-splattered fundus appearance [31] and foveal hypoplasia [32]. Females can in some rare instances (homozygous variant, partial monosomy skewed X-inactivation) display the same phenotypes as males [33].

\subsection{Negative clinical predictive value (probability not to develop the disease if the test is negative)}

Assume an increased risk based on family history for a nonaffected person. Allelic and locus heterogeneity may need to be considered.

Index case in that family had been tested:

If a non-affected relative is negative for the diseasecausing variant identified in the index case, it is highly predictive of unaffected status and the negative clinical predictive value will be close to $100 \%$. There is no increased risk other than a small risk related to the prevalence of other disease-causing genes or loci in the general population. Typically, the phenotype is evident in the first six months of life. Therefore, in an older non-affected relative this is not applicable.

Index case in that family had not been tested:

Unknown

\section{Clinical utility}

\section{1 (Differential) diagnostics: the tested person is clinically affected (to be answered if in 1.9 " $A$ " was marked)}

A number of syndromic conditions accompany albinism including Hermansky-Pudlak syndrome (HPS, OMIM 203300), Chediak-Higashi syndrome (CHS, OMIM 214500) and Griscelli syndrome (OMIM 214450). In these, the albinism phenotype is accompanied by bleeding disorders, immunodeficiency and organ manifestations. In the setting of a new patient, ruling out the syndromic forms are essential since it would alter the clinical care significantly from non-syndromic forms of albinism. Other differential diagnoses include SLC38A8 variants (FHONDA: foveal hypoplasia, optic nerve decussation defects and anterior segment dysgenesis, OMIM 609218), FRMD7 related infantile nystagmus (FIN, OMIM 310700) and PAX6 (OMIM 607108). In FRMD7 related infantile nystagmus, only grade 1 foveal hypoplasia has been reported with no evidence of hypopigmentation [34-36]. Recent work has shown that SLC38A8 mutations have a narrower but severe phenotypic spectrum (typically grade 3 or 4 foveal hypoplasia) compared to albinism (all grades of foveal hypoplasia seen) [34, 37]. Consistently in all affected patients with SLC38A8 mutations, the following are observed [37, 38]: (1) high-grade foveal hypoplasia (grade 3 or 4), (2) infantile nystagmus and (3) chiasmal misrouting detected on VEP. Anterior segment dysgenesis is likely a minor association or phenocopy. Thus, the only distinguishing phenotypical characteristic is the presence of ocular and/or cutaneous hypopigmentation in albinism.

\subsubsection{Can a diagnosis be made other than through a genetic test?}

No. (continue with 3.1.4)

Yes

$\begin{array}{ll}\bigotimes \text { (with caution) } & \\ \text { Clinically } & \square \\ \text { Imaging } & \square \\ \text { Endoscopy } & \square \\ \text { Biochemistry } & \square \\ \text { Electrophysiology } & \square \\ \text { Other (please describe): } & \square\end{array}$

Electrophysiological testing, including visually evoked potentials (VEPs) combined with evidence of foveal hypoplasia (using optical coherence tomography imaging) and a clinical presentation may allow for a diagnosis of OCA to be made with confidence. Recently, clinical diagnostic criteria have been proposed in which major criteria include foveal hypoplasia (grade 2 or more), VEP misrouting and ocular hypopigmentation. Further minor criteria include nystagmus, extraocular hypopigmentation, fundus hypopigmentation and grade 1 foveal hypoplasia [21]. However, there are often cases in which VEPs do not show the characteristic pattern in patients who may otherwise have positive molecular testing [21]. The authors report that these proposed criteria were met in up to $97.6 \%$ of patients with genetically confirmed albinism.

\subsubsection{Describe the burden of alternative diagnostic methods to the patient}

Patients with suspected albinism typically have abnormalities identified on slit-lamp examination, fundoscopy, optical coherence tomography (foveal hypoplasia) and VEP. Performing these investigations is often particularly difficult in infants and carries risk especially if some of these investigations have to be performed under sedation or anaesthesia. Identification of a variant affecting function in the albinism genes reduces the need for performing burdensome investigations. 
3.1.3 How is the cost-effectiveness of alternative diagnostic methods to be judged?

Due to the levels of cooperation required, often in a paediatric population repeated VEP testing may be required to achieve a reliable result [16], which may be deemed as inefficient in terms of cost. Additionally, MRI testing to rule out other significant pathology has cost implications of its own. Indeed, these investigations, even in combination with detailed ophthalmological examination may not yield a diagnosis in all cases.

3.1.4 Will disease management be influenced by the result of a genetic test?

No

Yes

Current treatments available are aimed at: (1) strategies to optimise vision (such as refractive error and amblyopia), (2) management of nystagmus and its sequelae (for example anomalous head posture) and (3) reducing glare [39]. However novel therapies addressing molecular errors in albinism are being developed [39].

\subsection{Predictive setting: the tested person is clinically unaffected but carries an increased risk based on family history}

(To be answered if in 1.9 "B" was marked)

\subsubsection{Will the result of a genetic test influence lifestyle and prevention?}

If the test result is positive (please describe)

If the test result is negative (please describe)

Not applicable

3.2.2 Which options in view of lifestyle and prevention does a person at-risk have if no genetic test has been done (please describe)?

Not applicable

\subsection{Genetic risk assessment in family members of a diseased person}

(To be answered if in 1.9 "C" $\mathrm{C}$ "was marked)
3.3.1 Does the result of a genetic test resolve the genetic situation in that family?

Yes. OCA is an autosomal recessive disorder, while OA is an X-linked disorder. Therefore, after molecular confirmation in the proband segregation can be assessed and carrier testing will help resolve the genetic situation in the family

3.3.2 Can a genetic test in the index patient save genetic or other tests in family members?

Identification of a variant affecting function in the index case could potentially obviate the need for further investigations such as electrodiagnostic testing in other affected family members.

3.3.3 Does a positive genetic test result in the index patient enable a predictive test in a family member?

Albinism typically manifests early in life; this includes development of nystagmus within six months of age. Therefore, performing genetic testing on a family member below six months of age might have predictive value.

\subsection{Prenatal diagnosis}

(To be answered if in 1.9 "D" was marked)

\subsubsection{Does a positive genetic test result in the index patient} enable a prenatal diagnosis?

Both prenatal and preimplantation genetic diagnosis are available for parents with a known variant affecting function.

\section{If applicable, further consequences of testing}

Please assume that the result of a genetic test has no immediate medical consequences. Is there any evidence that a genetic test is nevertheless useful for the patient or his/her relatives? (Please describe)

For syndromic cases in which life-threatening complications may occur, genetic testing may be paramount to confirming diagnosis. Conversely, confirmation of nonsyndromic forms of albinism will be helpful in alleviating anxiety. Genetic confirmation may forgo further unnecessary and cumbersome testing, decreasing burden on patients and healthcare providers. Persons with confirmed albinism may be more susceptible to damage from UV radiation, resulting in a higher risk for developing skin cancer [19]. 
This may require education regarding prevention strategies in the form of sun avoidance, barrier protection and sunscreen use. For patients with OCA who wish to be parents, genetic confirmation will allow for appropriate genetic counselling.

Acknowledgements This work was supported by EuroGentest2 (Unit 2: "Genetic testing as part of health care"), a Coordination Action under FP7 (Grant Agreement Number 261469) and the European Society of Human Genetics. We acknowledge the support of Fight for Sight, Academy of Medical Sciences, Ulverscroft Foundation, National Institute of Health Research and Medical Research Council. MGT is supported by the NIHR (CL-2017-11-003). MGT acknowledges the support of Fight for Sight, Ulverscroft Foundation, Medical Research Council, National Institute of Health Research (CL-2017-11003) and Academy of Medical Sciences.

\section{Compliance with ethical standards}

Conflict of interest The authors declare that they have no conflict of interest.

Publisher's note Springer Nature remains neutral with regard to jurisdictional claims in published maps and institutional affiliations.

\section{References}

1. Lasseaux E, Plaisant C, Michaud V, Pennamen P, Trimouille A, Gaston L, et al. Molecular characterization of a series of 990 index patients with albinism. Pigment Cell Melanoma Res. 2018:31:466-74.

2. Chaki M, Sengupta M, Mondal M, Bhattacharya A, Mallick S, Bhadra R, et al. Molecular and functional studies of tyrosinase variants among Indian oculocutaneous albinism type 1 patients. $\mathrm{J}$ Invest Dermatol. 2011;131:260-2.

3. Oetting WS, Pietsch J, Brott MJ, Savage S, Fryer JP, Gail Summers $\mathrm{C}$, et al. The R402Q tyrosinase variant does not cause autosomal recessive ocular albinism. Am J Med Genet Part A. 2009;149:466-9.

4. Norman CS, O'Gorman L, Gibson J, Pengelly RJ, Baralle DI, Ratnayaka JA, et al. Identification of a functionally significant triallelic genotype in the Tyrosinase gene (TYR) causing hypomorphic oculocutaneous albinism (OCA1B). Sci Rep. 2017;7:4415

5. Grønskov K, Jespersgaard C, Bruun GH, Harris P, BrøndumNielsen K, Andresen BS, et al. A pathogenic haplotype, common in Europeans, causes autosomal recessive albinism and uncovers missing heritability in OCA1. Sci Rep. 2019;9:1-7.

6. Monfermé S, Lasseaux E, Duncombe-Poulet C, Hamel C, Defoort-Dhellemmes S, Drumare I, et al. Mild form of oculocutaneous albinism type 1: phenotypic analysis of compound heterozygous patients with the R402Q variant of the TYR gene. Br J Ophthalmol. 2019;103:1239-47.

7. Luo D, Linpeng S, Zeng L, Tan H, Li Z, Wu L. Molecular genetic study of 59 Chinese Oculocutaneous albinism families. Eur J Med Genet. 2019;62:103709.

8. Yamada M, Sakai K, Hayashi M, Hozumi Y, Abe Y, Kawaguchi M, et al. Oculocutaneous albinism type 3: a Japanese girl with novel mutations in TYRP1 gene. J Dermatol Sci. 2011;64:217-22.

9. Straniero L, Rimoldi V, Soldà G, Mauri L, Manfredini E, Andreucci E, et al. Two novel splicing mutations in the SLC45A2 gene cause Oculocutaneous Albinism Type IV by unmasking cryptic splice sites. J Hum Genet. 2015;60:467-71.

10. Zhang Y, Zhang Y, Liu T, Bai D, Yang X, Li W, et al. Identification of two Chinese oculocutaneous albinism type 6 patients and mutation updates of the SLC 24A5 gene. J Dermatol. 2019;46:1027-30.

11. Khordadpoor-Deilamani F, Akbari MT, Karimipoor M, Javadi GR. Homozygosity mapping in albinism patients using a novel panel of 13 STR markers inside the nonsyndromic OCA genes: introducing 5 novel mutations. J Hum Genet. 2016;61:373-9.

12. Grønskov K, Dooley CM, Østergaard E, Kelsh RN, Hansen L, Levesque MP, et al. Mutations in C10orf11, a melanocytedifferentiation gene, cause autosomal-recessive albinism. Am J Hum Genet. 2013;92:415-21.

13. Lewis RA. Ocular Albinism, X-Linked. In: GeneReviews ${ }^{\circledR}$. Seattle: University of Washington; 1993. http://www.ncbi.nlm. nih.gov/pubmed/20301517.

14. Mayeur H, Roche O, Vêtu C, Jaliffa C, Marchant D, Dollfus H, et al. Eight previously unidentified mutations found in the OAI ocular albinism gene. BMC Med Genet. 2006;7:41.

15. Morice-Picard F, Lasseaux E, Plaisant C, Cailley D, Bouron J, Rooryck $\mathrm{C}$, et al. Albinism in a patient with mutations at both the OA1 and OCA3 loci. Pigment Cell Melanoma Res. 2016;29:107-9.

16. Campbell P, Ellingford JM, Parry NRA, Fletcher T, Ramsden SC, Gale $\mathrm{T}$, et al. Clinical and genetic variability in children with partial albinism. Sci Rep. 2019;9:16576.

17. Thomas MG, Maconachie GDE, Sheth V, McLean RJ, Gottlob I. Development and clinical utility of a novel diagnostic nystagmus gene panel using targeted next-generation sequencing. Eur J Hum Genet. 2017;25:725-34. http://www.nature.com/doifinder/10. 1038/ejhg.2017.44.

18. Zhang YZ, Bai DY, Qi Z, Zhao SZ, Yang XM, Li W, et al. Application of multiplex ligation-dependent probe amplification in the genetic testing of oculocutaneous albinism. Chi Med J. 2019;132:2011-2.

19. David CV. Oculocutaneous albinism. Cutis. 2013;91:E1.

20. Lund PM. Oculocutaneous albinism in southern Africa: population structure, health and genetic care. Ann. Hum. Biol. 2005;32:168-73.

21. Kruijt CC, de Wit GC, Bergen AA, Florijn RJ, Schalij-Delfos NE, van Genderen MM. The phenotypic spectrum of albinism. Ophthalmol. 2018;125:1953-60.

22. Rosenberg T, Schwartz M. X-linked ocular albinism: prevalence and mutations—a national study. Eur J Hum Genet. 1998;6:570-7.

23. Lewis RA. Oculocutaneous albinis type 1 . GeneReviews ${ }^{\circledR}$. Seattle: University of Washington; 2000. http://www.ncbi.nlm.nih. gov/pubmed/20301345.

24. Lewis RA. Oculocutaneous albinism type 2. GeneReviews ${ }^{\circledast}$. Seattle (WA): University of Washington; 2003. https://www.ncbi. nlm.nih.gov/books/NBK1232/.

25. Rooryck C, Morice-Picard F, Elçioglu NH, Lacombe D, Taieb A, Arveiler B. Molecular diagnosis of oculocutaneous albinism: new mutations in the OCA1-4 genes and practical aspects. Pigment Cell Melanoma Res. 2008;21:583-7.

26. Zhong Z, Gu L, Zheng X, Ma N, Wu Z, Duan J, et al. Comprehensive analysis of spectral distribution of a large cohort of Chinese patients with non-syndromic oculocutaneous albinism facilitates genetic diagnosis. Pigment Cell Melanoma Res. 2019;32:672-86.

27. Kausar T, Bhatti M, Ali M, Shaikh R, Ahmed Z. OCA5, a novel locus for non-syndromic oculocutaneous albinism, maps to chromosome 4q24. Clin. Genet. 2013;84:91-3.

28. Rosenmann A, Bejarano-Achache I, Eli D, Maftsir G, MizrahiMeissonnier L, Blumenfeld A. Prenatal molecular diagnosis of oculocutaneous albinism (OCA) in a large cohort of Israeli families. Prenat Diagn. 2009;29:939-46. 
29. Wei AH, Zang DJ, Zhang Z, Yang XM, Li W. Prenatal genotyping of four common oculocutaneous albinism genes in 51 Chinese families. J Genet Genomics. 2015;42:279-86.

30. Lenassi E, Clayton-Smith J, Douzgou S, Ramsden SC, Ingram S, Hall G, et al. Clinical utility of genetic testing in 201 preschool children with inherited eye disorders. Genet Med. 2020;22:745-51.

31. Charles SJ, Moore AT, Zhang Y, McMahon R, Barton DE, Yates $\mathrm{JR}$, et al. Carrier detection in $\mathrm{X}$ linked ocular albinism using linked DNA polymorphisms. Br J Ophthalmol. 1994;78:539-41.

32. Khan KN, Lord EC, Arno G, Islam F, Carss KJ, Raymond F, et al. Detailed retinal imaging in carriers of ocular albinism. Retin. 2018;38:620-8.

33. Pearce WG, Johnson GJ, Gillan JG. Nystagmus in a female carrier of ocular albinism. J Med Genet. 1972;9:126-8.

34. Thomas MG, Papageorgiou E, Kuht HJ, Gottlob I. Normal and abnormal foveal development. Br J Ophthalmol. 2020. https://doi. org/10.1136/bjophthalmol-2020-316348.
35. Thomas MG, Crosier M, Lindsay S, Kumar A, Araki M, Leroy BP, et al. Abnormal retinal development associated with FRMD7 mutations. Hum Mol Genet. 2014;23:4086-93.

36. Han J, Lee T, Lee JB, Han SH. Retinal microstructures are altered in patients with idiopathic infantile nystagmus. Graefe's Arch Clin Exp Ophthalmol. 2017;255:1661-8.

37. Kuht HJ, Han J, Maconachie GDE, Park SE, Lee ST, McLean R, et al. SLC38A8 mutations result in arrested retinal development with loss of cone photoreceptor specialization. Hum Mol Genet. 2020;29:2989-3002.

38. Poulter JA, Al-Araimi M, Conte I, Van Genderen MM, Sheridan E, Carr IM, et al. Recessive mutations in SLC38a8 cause foveal hypoplasia and optic nerve misrouting without albinism. Am J Hum Genet. 2013;93:1143-50.

39. Liu S, Kuht HJ, Moon EH, Maconachie GDE, Thomas MG. Current and emerging treatments for albinism. Surv Ophthalmol. 2020;S0039-6257:30145-4. https://doi.org/10.1016/j.survophthal. 2020.10.007. [Online ahead of print]. 